\title{
EVOLUÇÃO DOS SUBSTITUTOS DE GORDURA UTILIZADOS NA TECNOLOGIA DE ALIMENTOS
}

\author{
CRISTIANE S. MONTEIRO* \\ SOLANGE TERESINHA CARPES* \\ VANESSA H. KALLUF** \\ DANIELLE SELL DYMINSKI ${ }^{* * *}$ \\ LYS MARY BILESKI CÂNDIDO****
}

\begin{abstract}
O objetivo deste trabalho foi reunir informações atualizadas sobre os substitutos de gordura, abrangendo a diversidade desses ingredientes e seu potencial de aplicação. Foram abordados os substitutos de gordura derivados de carboidratos, de lipídios e de proteínas, bem como a combinação dessas três categorias. Verificou-se que os substitutos de gordura podem ser facilmente incorporados em muitos alimentos como, laticínios, queijos, bebidas, produtos de panificação, chocolates, molhos para saladas, maioneses e sobremesas. A mais nova geração de substitutos de gordura tem incentivado a revolução dos alimentos com baixo conteúdo calórico, devido principalmente à demanda proveniente de consumidores preocupados com a saúde. Essa demanda tem permitido o avanço tecnológico e muitas melhorias estão sendo efetuadas com relação à funcionalidade e a palatabilidade desses produtos.
\end{abstract}

PALAVRAS-CHAVE: SUBSTITUTO DE GORDURA; ALIMENTOS FUNCIONAIS.

Doutorandas do Programa de Pós-Graduação em Tecnologia de Alimentos, Universidade Federal do Paraná (UFPR), Curitiba-PR.

** Mestre em Tecnologia de Alimentos pela UFPR, Curitiba-PR.

*** Doutora em Tecnologia de Alimentos pela UFPR, Curitiba-PR.

**** Professora, Doutora, Programa de Pós-Graduação em Tecnologia de Alimentos, UFPR, Curitiba-PR (e-mail: lysmary@ufpr.br). 


\section{INTRODUÇÃO}

A partir dos anos oitenta, a população vem modificando seus hábitos alimentares visando principalmente a redução da ingestão de açúcar e de gordura. Segundo DREWNOWSKI (1990), as pessoas estão cada vez mais informadas quanto à necessidade de reduzir as gorduras de sua alimentação.

O lançamento de novos produtos no mercado tem sido incentivado pela divulgação de trabalhos sobre a correta ingestão de nutrientes para a manutenção da saúde (SILVIERI e OLIVEIRA, 2002; KRÜCKENPEREIRA, COSTA e BOLZAN, 2002; FAT..., 2006) e pelo interesse dos consumidores por alimentos saudáveis. $O$ desenvolvimento de produtos com baixo teor de gordura, baixas calorias e baixo colesterol são algumas tendências na indústria de alimentos.

A gordura constitui ingrediente importante para os aspectos sensoriais e tecnológicos dos alimentos. Está associada com a percepção do aroma, da cremosidade, do sabor e com a sensação de saciedade após as refeições (NEY, 1988). Importantes compostos como, ácidos graxos essenciais, drogas lipofílicas, precursores de prostaglandinas e as vitaminas lipossolúveis $A, D, E$ e $K$ são de natureza lipídica (SLESINSKI, SUBAR e KAHLE, 1995).

A produção de alimentos com baixo teor de gordura demanda a utilização de produtos com os atributos dos lipídios, mas com conteúdo calórico reduzido. Podem ser empregados estabilizantes, emulsificantes, espessantes e outros aditivos com propriedades específicas. Sua produção é difícil e nem sempre se consegue expressiva redução calórica em função da manutenção das propriedades sensoriais.

O substituto de gordura ideal seria um composto seguro que apresentasse todas as propriedades funcionais das gorduras, mas com baixo teor calórico. Infelizmente esse composto não existe, embora estejam disponíveis no mercado diversos produtos com algumas dessas propriedades e que na correta combinação e proporção permitem o desenvolvimento de grande número de produtos alimentícios (CÂNDIDO e CAMPOS, 1996; ADA, 2005). 
Os substitutos de gorduras podem melhorar a qualidade de muitos produtos novos com teor de gordura reduzido e, quando usados nas dosagens corretas, servir para o crescimento do mercado e não apenas como opção para dietas. Alguns substitutos de gordura podem ser facilmente incorporados em muitos alimentos como produtos de laticínios, queijos e bebidas, molhos para saladas, maioneses e sobremesas (AKOH, 1996, FAT..., 2006).

O objetivo deste trabalho foi reunir informações atualizadas sobre os substitutos de gorduras, abrangendo a diversidade desses ingredientes e seu potencial de aplicação.

\section{SUBSTITUTOS DE GORDURAS}

\subsection{CONCEITOS E CLASSIFICAÇÃO}

Os substitutos de gorduras podem ser classificados, segundo a American Dietetic Association (ADA, 2005), como fat substitutes, fat analogs, fat extenders e fat mimetics. Fat substitutes são ingredientes que se assemelham às gorduras e óleos convencionais e podem substituí-los completamente. São produzidos a base de gorduras e muitas vezes estáveis nas temperaturas de cozimento e de frituras. Fat analogs são compostos com muitas características da gordura, mas podem alterar a digestibilidade e o valor nutricional do alimento. Fat extenders otimizam a funcionalidade da gordura existente no alimento, permitindo o uso de menor quantidade de gordura no produto. Fat mimetics são ingredientes que imitam uma ou mais funções sensoriais e físicas da gordura no alimento. Elaborados a base de carboidratos, proteínas, ou componentes de gorduras podem ser usados isoladamente ou em combinação, fornecendo de zero a nove $\mathrm{kcal} / \mathrm{g}$. Não devem ser empregados em frituras, pois absorvem água e estão sujeitos a sofrer escurecimento em função do calor elevado.

Os substitutos de gorduras são produzidos a base de proteínas, carboidratos e lipídios, ou de sua combinação (CÂNDIDO e CAMPOS, 1996; FAT..., 2006). A utilização do substituto de gordura depende do tipo de alimento como mostra o Quadro 1. 


\section{QUADRO 1 -EXEMPLO DE SUBSTITUTOS DE GORDURA EM VÁ- RIAS CATEGORIAS}

\begin{tabular}{|c|c|c|c|}
\hline \multirow[t]{2}{*}{ Alimentos } & \multicolumn{3}{|c|}{ Substitutos de gordura } \\
\hline & Carboidratos & Proteínas & Gorduras \\
\hline $\begin{array}{l}\text { Produtos de } \\
\text { panificação }\end{array}$ & $\begin{array}{l}\text { Fibras, gomas, inulina, amidos } \\
\text { maltodextrina e polidextroses }\end{array}$ & $\begin{array}{l}\text { Proteína microparticulada, } \\
\text { proteína concentrada de } \\
\text { soro modificado e misturas } \\
\text { de proteínas }\end{array}$ & $\begin{array}{l}\text { Benefat }^{\circledR} \mathrm{e} \\
\text { emulsificantes }\end{array}$ \\
\hline $\begin{array}{l}\text { Produtos a base de } \\
\text { cereais e grãos }\end{array}$ & $\begin{array}{l}\text { Fibras, gomas, inulina, amidos, } \\
\text { maltodextrina, polidextrose }\end{array}$ & Proteína microparticulada & $\begin{array}{l}\text { Emulsificantes } \\
\text { e Olean }\end{array}$ \\
\hline Doces, balas & $\begin{array}{l}\text { Celulose, gomas, inulina, oatrim, } \\
\text { maltodextrina, polidextrose e } \\
\text { amidos }\end{array}$ & Proteína microparticulada & $\begin{array}{l}\text { Benefat }^{\circledR} \\
\text { Caprenin }^{\circledR}, e \\
\text { emulsificantes }\end{array}$ \\
\hline Óleos & NA & Proteína microparticulada & NA \\
\hline Produtos lácteos & $\begin{array}{l}\text { Celulose, gomas, inulina, amidos, } \\
\text { maltodextrina, oatrim, polidextrose }\end{array}$ & $\begin{array}{l}\text { Proteína microparticulada, } \\
\text { proteína concentrada de } \\
\text { soro modificado }\end{array}$ & Emulsificantes \\
\hline $\begin{array}{l}\text { Sobremesas } \\
\text { congeladas e } \\
\text { refrigerados }\end{array}$ & $\begin{array}{l}\text { Celulose,gomas, inulina, oatrim, } \\
\text { maltodextrina, polidextrose e } \\
\text { amidos }\end{array}$ & $\begin{array}{l}\text { Proteína microparticulada, } \\
\text { proteína concentrada de } \\
\text { soro modificado e misturas } \\
\text { de proteínas }\end{array}$ & $\begin{array}{l}\text { Benefat }{ }^{\circledR} \mathrm{e} \\
\text { emulsificantes }\end{array}$ \\
\hline $\begin{array}{l}\text { Produtos a base de } \\
\text { carne e de aves }\end{array}$ & $\begin{array}{l}\text { Gomas, inulina, maltodextrina, } \\
\text { oatrim e amidos }\end{array}$ & NA & NA \\
\hline $\begin{array}{l}\text { Sopas, milhos e } \\
\text { "gravies" }\end{array}$ & $\begin{array}{l}\text { Celulose, gomas, inulina, oatrim, } \\
\text { maltodextrina e amidos }\end{array}$ & $\begin{array}{l}\text { Proteína microparticulada, } \\
\text { proteína concentrada de } \\
\text { soro modificado }\end{array}$ & Emulsificantes \\
\hline $\begin{array}{l}\text { Salgadinhos } \\
\text { aromatizados }\end{array}$ & $\begin{array}{l}\text { Celulose, fibras, gomas, amidos e } \\
\text { maltodextrina }\end{array}$ & NA & Olean $^{\circledR}$ \\
\hline
\end{tabular}

$\mathrm{NA}=$ Não-Aplicável

Fonte: ADA, 2005.

\subsection{SUBSTITUTOS DE GORDURADERIVADOS DE CARBOIDRATOS}

Estão disponíveis no mercado substitutos de gordura derivados de celulose, dextrinas, fibras, gomas, amido, inulina e outros.

Celulose (Avicel ${ }^{\circledR}$ cellulose gel, MethocelTM, Solka-Floc $\circledast$, Just fiber) é utilizada em produtos de laticínios, molhos, sobremesas geladas e temperos para saladas. A celulose, ingrediente não-calórico, é produzida como material microparticulado que dispersado no alimento se assemelha à fase gordurosa (IMESON, 1997). Retêm a umidade, age na textura e como estabilizante, além de modificar a viscosidade 
em emulsões. Proporciona sabor e o fluxo de gordura, mas não o sabor característico de gordura (TORRES, 2002).

Os Amidos e Amidos Modificados foram criados e idealizados para uso em grande variedade de produtos de panificação como substitutos de gorduras. Melhoram a aparência, o sabor, a textura, a vida útil e o valor nutricional de bolos, cookies, recheios, pães, e outros (LUNARDINI, 2005). Comercialmente estão disponíveis produtos como Frigex-NS, Gel "N" Creamy-NS, Instant Purê Flo-NS, Instant Stellar" ${ }^{\mathrm{TM}}$, Instant W-11-AMP, Leanbind-NS, Amalean ${ }^{\circledR}$, Farinex ${ }^{\mathrm{TM}}$, VA15, VA20, $\mathrm{N}$-Lite, OptaGrade ${ }^{\circledR}$, Perfectamyl $I^{\mathrm{TM}} \mathrm{AC}, \mathrm{AX}-1, \mathrm{AX}-2$, Pure-Gel ${ }^{\circledR}$, Mirathick, Remyline-RI, Remygel-RI, Stenderlean-NS, Starch Plus SPRCNP, Sta-lim ${ }^{\mathrm{TM}}$ e Inscosity ${ }^{\mathrm{R}}$, entre outros. Geralmente são produzidos a partir de batata, milho, aveia, trigo, mandioca e arroz. Os amidos fornecem de 1 a $4 \mathrm{kcal} / \mathrm{g}$ e são utilizados como substitutos de gordura, estabilizantes, espessantes, gelificantes e emulsificantes. Atuam como agente de corpo e modificadores de textura. Também são usados em carnes processadas, recheios de pães, molhos, sobremesas congeladas, temperos e derivados de leite (CEREDA e VILPOUX, 2003; TORRES, 2002; DELCOUR e EERLINGER, 1996).

PASELLI BC, substituto de gordura, é produzido a base de amido de batata modificado enzimaticamente. Além de solúvel em água fria é estável ao pH e em baixas temperaturas. Esse tipo de amido apresenta habilidade para interagir com lipídios e especialmente com emulsificantes, resultando num produto com propriedade geleificante que pode ser usado em maioneses, sobremesas geladas, molhos e coberturas (ZAMBRANO e CAMARGO, 2002; BYRNE, 1992; CÂNDIDO e CAMPOS, 1996).

MIRA-THIK, elaborado a base de milho, apresenta a capacidade de produzir géis rapidamente. Fornece viscosidade e transparência ideal para produtos como molhos e misturas, sem a necessidade do uso de altas temperaturas (CEREDA e VILPOUX, 2003).

Dextrinas (Amylum, $\mathrm{N}-\mathrm{Oil}{ }^{\circledR}$ ) podem substituir todas ou algumas gorduras em grande variedade de produtos, fornecendo $4 \mathrm{kcal} / \mathrm{g}$ de produto. Entre as fontes de dextrina está a tapioca. As dextrinas são utilizadas em molhos, saladas, sobremesa, produtos de laticínios e sobremesas geladas (FAT..., 1996). 
Maltodextrinas (CrystaLean ${ }^{\circledR}$, Lorelite, Lycadex ${ }^{\circledR}$, Maltrin $^{\circledR}$, Paselli ${ }^{\circledR}$, D-lite, Paselli ${ }^{\circledR}$ Excel, Paselli ${ }^{\circledR}$ SA2, Star-dri ${ }^{\circledR}$, Rice-Trim) fornecem 4 $\mathrm{kcal} / \mathrm{g}$ de produto. Elaboradas a partir do milho, trigo, batata e mandioca são utilizadas como substitutos de gordura, agentes de corpo e modificadores de textura em produtos de panificação, laticínios, molhos, cremes, embutidos, sobremesas geladas, extrusados e bebidas (IFT, 1998).

Fibras (Opta ${ }^{\top \mathrm{M}}$, Oat Fiber, Snowite, Ultracel ${ }^{\mathrm{TM}}$, Z-Trim ${ }^{\circledR}$, WonderSlim ${ }^{\circledR}$, Betatrim $^{\circledR}$, Grindsted ${ }^{\circledR}$, Splendid ${ }^{\circledR}$ ) podem fornecer integridade estrutural, volume, capacidade de homogeneização, adesividade e estabilidade de prateleira em produtos com gordura reduzida. Suas aplicações incluem assados, carnes e produtos extrusados (FANTA, 1995; LEE et al., 2005; LEE, INGLETTI e CARRIERE, 2004).

O enriquecimento de pães com fibras pode ser conseguido com o derivado da polpa de citrus CITRI-FI ${ }^{(\mathrm{TM})}$ da Fiberstar Inc. Essa fibra natural de citrus fornece mais de $74 \%$ da fibra alimentar total necessária na dieta humana. A fibra inibe a migração da umidade, tendo como resultado a distribuição mais uniforme da umidade em massas congeladas de pães e em produtos frescos. Essa propriedade (impedir a migração da umidade) melhora as características de frescor, gosto e aroma da crosta em pães convencionais e em pães com elevado teor de fibra durante período prolongado de armazenagem (BERRY, 2004).

Z-TRIM, empregado em queijos e hambúrgueres, é elaborado a base de casca de aveia, casca de arroz, vagem de soja, ervilhas e farelo de milho ou trigo (INGLETT, 1996). As cascas ou farelo, processados em fragmentos microscópicos e purificados, são secos e moídos na forma de pó fino. Ao absorver água, o pó se expande para formar gel que fornece alimentos com textura agradavelmente macia. Produzido a partir de fibras naturais, Z-TRIM não agride o sistema digestivo quando consumido em quantidades normais (INGLETT, 1996).

NUTRIM X, produzido a base de aveia ou grãos de cevada, é rico em beta-glucana, reponsável pela redução de fatores de risco de doenças cardiovasculares. Tem sido utilizado em produtos de panificação, leite, queijo, sorvetes e refrigerantes (THEBAUDIN e LEFEBEVRE, 1997; LEE et al., 2005). 
OATRIM, também conhecido como TRIMCHOICE ${ }^{\mathrm{TM}}$, é rico em betaglucana. Elaborado a base de aveia pode ser facilmente incorporado em muitos alimentos como produtos lácteos, bebidas, temperos para saladas, molhos e manteiga de amendoim (ESKIN e FANTA, 1995). Os substitutos de gordura baseados na aveia oferecem excelente textura e aparência, melhorando a viscosidade dos alimentos. Os produtos de panificação podem ser beneficiados com a substituição da gordura na faixa de $25 \%$ até $100 \%$ em misturas para bolos, biscoitos, croissants e muffins (DUXBURY, 1993).

OATVANTAGETM (Glanbia Nutritionals), fibra solúvel da aveia superconcentrada, é versatil e 100\% natural. Rica em beta-glucana (54\%) tem sido recomendada por reduzir os níveis de colesterol sérico, notavelmente o colesterol prejudicial à saúde (LDL). A beta-glucana retarda a absorção de glucose sanguínea, modulando os níveis de insulina e de glucose. O consumo desse polissacarídio pode resultar na redução da fome e/ou aumento da saciedade. Dietas com aveia também reduzem a pressão sanguínea. OATVANTAGETM pode ser usado com sucesso em alimentos, bebidas e como suplemento alimentar (1,4 $\mathrm{g}$ do produto fornecem $0,75 \mathrm{~g}$ de beta-glucana da aveia, quantidade requerida para as declarações de propriedades de saúde na rotulagem). Uma dose de duas cápsulas, ou 1,4 g de OatVantage ${ }^{\mathrm{TM}}$ em $200 \mathrm{~mL}$ de suco de laranja, oferece os mesmos benefícios à saúde do coração que $25 \mathrm{~g}$ da aveia in natura (DAVIS, 2005).

As gomas (KELCOGEL ${ }^{\circledR}, \mathrm{KELTROL}^{\circledR}, \mathrm{KELGUM}^{\circledR}$, Slendid $^{\mathrm{TM}}$ ) são conhecidas como colóides hidrofílicos, ou hidrocolóides com propriedades espessantes e geleificantes. Nesse grupo estão goma guar, goma arábica, goma de alfarroba, goma xantana, carregenatos e pectinas. Não-calóricas, são utilizadas em molhos e saladas, sobremesas e embutidos (WILLIANS, 2000).

PUREGLUCAN, também conhecido como Curdlan, é produzido por um microorganismo mediante fermentação da glicose. Pode formar dois tipos de géis induzidos pelo aquecimento. Os géis, estáveis em altas e baixas temperaturas, diferenciam-se pela sua reversibilidade térmica (KITAMURA, 2005). É utilizado como agente de textura que apresenta quantidade extremamente baixa de gordura. $O$ PUREGLUCAN pode substituir a gordura em produtos lácteos gelados (LERNER, 1997). 
Inulina (Raftiline ${ }^{\circledR}$, Fruitafit ${ }^{\circledR}$, Fibruline ${ }^{\circledR}$ ) apresenta reduzido teor calórico (de 1 a $1,2 \mathrm{kcal} / \mathrm{g}$ ) e pode ser utilizada como substituto de gordura, de açúcar e como fonte de fibra solúvel. Extraída da raíz da chicória é utilizada em iogurtes, queijos, sobremesas geladas, produtos panificados, recheios e embutidos (PASSOS e PARK, 2003).

Polióis constituem grupo de adoçantes que substituem o açúcar $(1,6$ a $3,0 \mathrm{kcal} / \mathrm{g}$, dependendo do poliol). Pela sua plasticidade e propriedades umectantes, os polióis também podem ser utilizados como substitutos de gordura em produtos com baixa ou nenhuma gordura (FANTA, 1995).

Polidextrose $\left(\right.$ Litesse $^{\circledR}$, Sta-Lite $^{\mathrm{TM}}$, Gelcarin $\left.{ }^{\circledR}\right)$ fornece $1 \mathrm{kcal} / \mathrm{g}$ e também é utilizada como agente de corpo em diversos produtos, incluindo assados, goma de mascar, confeitos, coberturas, sobremesas lácteas e pudins. A polidextrose, polímero hidrossolúvel da dextrose, contém pequenas quantidades de sorbitol e ácido cítrico (FANTA, 1995).

\subsection{SUBSTITUTOS DE GORDURA DERIVADOS DE LIPÍDIOS}

Os substitutos de gordura derivados de lipídios encontrados no mercado são SALATRIM, OLESTRA, BETAPOL, BOB, EMULSIFICANTES, GLICEROL PROPOXILADO ESTERIFICADO (EPG) e SORBESTRIN.

Emulsificantes (Dur-Lo $\left.{ }^{\circledR}, \mathrm{EC}^{\mathrm{TM}}-25\right)$ incluem óleos vegetais, mono e diacilgliceróis emulsificantes, que podem juntamente com a água substituir toda ou parte da gordura em bolos, cookies, coberturas e produtos derivados do leite. Apesar de fornecerem $9 \mathrm{kcal} / \mathrm{g}$ são utilizados em menor quantidade que as gorduras, resultando em redução no teor total de gordura do produto final.

Glicerol Propoxilado Esterificado (EPG) forma-se pela reação do glicerol com óxido de etileno e posterior esterificação com ácidos graxos. Tem sido aplicado em sobremesas geladas, panificação, cremes, molhos, frituras e cookies.

SORBESTRIN, estável em altas temperaturas, fornece aproximadamente $1,5 \mathrm{kcal} / \mathrm{g}$. Esse produto, composto por ésteres de ácidos graxos de sorbitol e anidridos de sorbitol é utilizado em 
maioneses, panificação, molhos, e também para frituras.

SALATRIM (Benefat ${ }^{\circledR} ;$ Caprenin $^{\circledR}$ ) contém $5 \mathrm{kcal} / \mathrm{g}$ e reduz em $55 \%$ o valor calórico da gordura original (9 kcal/g) (LERNER, 1997). Trata-se de gordura composta por glicerol e pelos ácidos acético, propiônico, butírico e esteárico. O triacilglicerol resultante substitui certos tipos de gorduras, mas não pode ser empregado para frituras. Esse substituto pode ser utilizado em produtos de confeitaria, chocolates, biscoitos, derivados de leite, recheios, margarinas, assados e molhos (TORRES, 2002).

OLESTRA (Olean ${ }^{\circledR}$ ), ou poliéster de sacarose, é obtido da síntese de ácidos graxos saturados e insaturados de óleos vegetais. Forma-se pela mistura de hexaésteres, heptaésteres e octaésteres de sacarose com ácidos graxos, cujo número de carbonos varia de 8 a 18 (UIEARA, 2000). O Olestra não é absorvido e nem metabolizado pelo organismo, podendo causar problemas digestivos (TORRES, 2002). Termoestável, pode ser empregado em frituras. Esse ingrediente proporciona sabor, textura e cor ao alimento, além de aumentar sua vida-de-prateleira. Foi aprovado como aditivo alimentar para aromatização de snacks e cookies pela Food and Drug Administration (FDA) e apresenta potencial para outras aplicações (UIEARA, 2000; BUSETTI, 1995).

BETAPOL, produzido via interesterificação enzimática, baseia-se em gorduras vegetais com a finalidade de simular a distribuição de ácidos graxos da gordura do leite humano. Esse triacilglicerol só pode ser obtido com lipase 1,3 específica, mediante reação de tripalmitina com ácidos graxos insaturados (D'AGOSTINI, 2001). O produto formado é rico em palmitato na posição 2 e de resíduos de ácidos graxos insaturados nas posições 1,3 . O produto está sendo desenvolvido industrialmente para uso em formulações infantis (SANDERS, OMES e EARL 2001).

BOENINA (BOB) tem sido produzido no Japão por interesterificação enzimática entre trioleína e ácido beênico (C22:0). É utilizado como substituto de gordura em produtos de cacau (GIOILLI, 2002) por inibir o fat bloom quando adicionado ao chocolate (D'AGOSTINI, 2001; GUNSTONE, 1998). 


\subsection{SUBSTITUTOS DE GORDURA DERIVADOS DE PROTEÍNAS}

Proteína Microparticulada (Simplesse ${ }^{\circledR}$ ), Proteína Concentrada de Soro Modificado (Dairy-Lo ${ }^{\circledR}$, super creme, Calpro, AMP800, Ultra creme, Nutrilac) e outros (K-Blazer ${ }^{\circledR}$, Ultra-bake ${ }^{\mathrm{TM}}$, Ultra-freeze ${ }^{\mathrm{TM}}$, Lita $^{\circledR}$ ) são exemplos de substitutos de gordura derivados de proteínas encontrados no mercado.

Proteína Microparticulada (Simplesse ${ }^{\circledR}$ ) apresenta valor calórico reduzido (1-2 kcal/g), sendo obtida a partir do soro do leite ou leite e ovos. Tem sido empregada em queijos, iogurtes, margarinas, molhos, sopas, cremes, maionese e produtos de panificação.

Proteína Concentrada de Soro Modificada (Dairy-Lo ${ }^{\circledR}$, Super creme, Calpro, AMP800, Ultra-creme, Nutrilac), obtida pela inativação térmica controlada do soro do leite, tem sido utilizada em sorvetes, congelados, molhos, produtos de laticínios e produtos de panificação.

K-Blazer ${ }^{\circledR}$, Ultra-bake ${ }^{T M}$, Ultra-freeze ${ }^{\top \mathrm{M}}$ e Lita $^{\circledR}$ são similares aos substitutos de gordura a base de proteínas microparticuladas (clara de ovo e proteínas de leite e milho). Fornecem de 1 a $4 \mathrm{kcal} / \mathrm{g}$ e são estáveis em produtos assados e em sobremesas geladas, mas não em frituras (NITZKE, TANUMIHARDJO e RETTAMMEL, 2004; SANDROU e ARVANITOYANNIS, 2000).

\subsection{COMBINAÇÃO DE SUBSTITUTOS DE GORDURAS}

É possível encontrar substitutos de gorduras a partir da combinação das três bases (carboidratos, proteínas e lipídios). Essa combinação melhora atributos funcionais dos produtos como, textura, sabor e sensação tátil bucal da gordura ("mouthfeel"). Entretanto esses substitutos retêm água impossibilitando seu uso em frituras.

OptaMax ${ }^{\circledR}$ e Fantesk ${ }^{\circledR}$ são exemplos de substitutos de gordura combinando Carboidrato+Lipídios e $\mathrm{Mimix}^{\circledR}$ da combinação de Carboidrato+Proteína. Segundo a ADA (2005), o substituto de gordura Mimix ${ }^{\circledR}$ ainda está em avaliação e não disponível no mercado.

Substituto de gordura com mistura de amido, minúsculas gotas de óleo e água, o FANTESK é empregado em produtos cárneos, sorvetes 
e margarinas (ESKIN e FANTA, 1995). LIQUID FANTESK ${ }^{\text {TM }}$, suspensão estável de gotículas de óleo microscópicas em solução de amido ou gel, tem a aparência de produto a base de amido com a propriedade da fase oleosa incluída. O LIQUID FANTESK ${ }^{\mathrm{TM}}$ pode ser seco em flocos ou moído na forma de pó muito fino não-oleoso. O óleo está contido como microgotículas dentro do pó seco de amido. O pó FANTESK ${ }^{\mathrm{TM}}$ pode ser usado diretamente como ingrediente seco ou ser facilmente misturado com água quente para reconstituir o produto líquido original em muitas concentrações de sólidos (ESKIN e FANTA, 1995).

\section{CONSIDERAÇÕES FINAIS}

A mais nova geração de substitutos de gordura tem incentivado a revolução dos alimentos com baixo conteúdo calórico, devido principalmente à demanda proveniente de consumidores preocupados com a saúde. Essa demanda tem permitido o avanço tecnológico e muitas melhorias estão sendo efetuadas com relação à funcionalidade e a palatabilidade desses produtos.

\section{Abstract \\ EVOLUTION OF FAT REPLACERS UTILIZED IN FOOD TECHNOLOGY}

The aim of this work was to gather up-to-date informations on fat replacers including the diversity of those ingredients and its application potential. The fat replacers derived from carboydrates, lipids and proteins were approached, as well as the combination of those three categories. It was verified that the fat replacers can be easily incorporated in many foods as, dairy products, cheeses, beverages, breads, chocolates, sauces for salads, mayonnaises and desserts. The newest generation of fat replacers has been motivating the revolution of the foods with low caloric content, owed mainly to the consumers' demand worried with health. That demand has been allowing the technological progress and a lot of improvements are being made with relationship to the functionality and the palatability of those products. 
KEY-WORDS: FAT REPLACER; FUNCTIONAL FOODS.

\section{REFERÊNCIAS}

1 ADA. American Dietetic Association. Position of the American Dietetic Association: fat replacers. Journal of American Dietetic Association, n.105, p.266-275, Fev. 2005.

$2 \mathrm{AKOH}, \mathrm{C} . \mathrm{C}$. Fat replacer. Food Technology, Chicago, v.52, p.4753, 1998.

3 BERRY, D. Breads on the rise. On line: http:// www.foodproductdesign.com/archive/2004/1004DE.html. Acesso em: 22 jun. 2005.

4 BUSETTI, M. Building better reduce-fat baked goods and snacks. Prepared Foods, v.8, n.164, p.77-78, 1995.

5 BYRNE, M. Fat replacers in focus. Chilton's Food Engineering International, Radnor, v. 17, n. 4, p. 4148, Sept. 1992.

6 CÂNDIDO, L. M. B. ; CAMPOS, A. M. Substitutos de gorduras. Boletim do CEPPA, v. 13, n.2, p. 125-164, jul./dez. 1996.

7 CEREDA, M. P.; VILPOUX, O, F. Tecnologia, usos e potencialidades de tuberosas amiláceas latino americanas. São Paulo: Fundação Cargill, 2003. 711 p.

8 D'AGOSTINI, D. Obtenção de lipídeos estruturados interesterificação de triacil gliceróis de cadeia média e longa. São Paulo, 2001. 185 p. Tese (Doutorado em Tecnologia Bioquímica Farmacêutica), Área de Concentração em Tecnologia de Alimentos, Universidade de São Paulo.

9 DAVIS, R. H. OatVantage proves superiority in building cholesterol-lowering viscosity. 2005-04-26 - Nurture, Inc. On line: http://www.oatvantage.com. Acesso em: 23 jun. 2005.

10 DELCOUR, J.A; EERLINGER, R.C. Analytical implications of the 
classification of resistant start as dietary fiber. Cereal Foods World, St. Paul, v.41,n.2,p.85-86,1996.

11 DREWNOWSKI, A. The new fat replacement: a strategy for reducing fat consumption. J. Postgraduate Medicine, v.87,p.111121,1990 .

12 DUXBURY, D.D. Fat substitute with fiber now commercialized (TrimChoice). Food Processing, March 01, 1993. On line: http:/ /static.highbeam.com/f/foodprocessing. Acesso em: 05 nov. 2004.

13 ESKIN, K. FANTA, G.F. Heart-Healthy Foods. Cereal Products \& Food Science Research, National Center for Agricultural Utilization Research (NCAUR), 1995. On line: http:// www.ncaur.usda.gov/cpf/trimtech.html. Acesso em: 08 nov. 2004.

14 FANTA, G.F. Yes, You can mix oil and water. Food \& Nutrition Research Briefs, Agricultural Research Service (ARS), 1995. On line: http://www.ars.usda.gov/is/np/fnrb/fnrb1095.html. Acesso em: 05 nov. 2004.

15 FAT replacers: food ingredients for healthy eating. 2006. On line: http://www.caloriecontrol.org/fatrepl.html. Acesso em: 18 ago. 2006.

16 GRUNWALD, G. Como desenvolver e lançar um novo produto no mercado. São Paulo: Makron Besks, 1993. 550 p.

17 GIOILLI, L.A. Lipídios estruturados. In: CURI, R.; POMPÉIA, C.; MIYASAKA, C.K.; PROCÓPIO, J. Entendendo a gordura: os ácidos graxos. São Paulo: Manole, 2002.cap.33, p.457-465.

18 GUNSTONE, F. D. Movements towards tailor-made fats. Progress in Lipid Research, v.37, n.5, p.277-305, 1998.

19 INGLETT, G. E. New trim from trash and more. Food \& Nutrition Research Briefs, Agricultural Research Service (ARS), 1996. On line: http://www.ars.usda.gov/is/np/fnrb/fnrb1096.html. Acesso em: 08 nov. 2004. 
20 IMESON, A. Thickening and gelling agents for food. New York: Chapman \& Hall, 1997. 336 p.

21 IFT. Institute of Food Technologists. Fat replacers. Food Technology, v.52, n.3, p.47-53, 1998.

22 KITAMURA, Y. Export products list. Takeda-Kirin Foods Corporation. On line: http://www.takeda-kirin.co.jp/english/. Acesso em: 22 set. 2005.

23 KRÜCKEN-PEREIRA, L. ; COSTA, M. D.; BOLZAN, A. Gestão do conhecimento aplicada ao desenvolvimento de novos produtos. Revista Inteligência Empresarial, n. 12, p.48-56, jul. 2002.

24 LEE, S.; KIM, S.; INGLETT, G.E.; CARRIERE, C.J. Effect of Nutrim and Oatrim on the physical rheological properties of cakes. Disponível em: http://www.ars.usda.gov/pandp/people/ people.htm. Acesso em: 19 dez. 2005.

25 LEE, S.; INGLETT, G.E.; CARRIERE, C.J. Effect of Nutrim and Flaxseeds on the physical rheological properties of cakes. Cereal Chemystry, v. 81, n.5. p.637-642, 2004.

26 LERNER, M. Removing the fat. Chemical Market Reporter. Jun 16, 1997. Disponível em: http://www.highbeam.com Acesso em: 16 ago. de 2006.

27 NARDINI, A. C. Como melhorar a textura, sabor e performance de produtos em panificação. ABAM, v. 3, n.11, 2005. Disponível em: http://www.abam.com.br/revista/revista11/textura.php. Acesso em: 16 ago. de 2005.

$28 \mathrm{NEY}, \mathrm{K} . \mathrm{H}$. Sensogamme, eine methodische Erwiterung der Aromagramme. Gondian, v. 88, n. 1, p. 19-29, 1988.

29 NITZKE, S.; TANUMIHARDJO, S.; RETTAMMEL, A. Nutrition for family living. March, 2004. Disponível em: www.uwex.edu/ $\mathrm{ces} / \mathrm{wnep} / \mathrm{specialist} / \mathrm{hfl} / \mathrm{mmpdfs} / 0403$.pdf. Acesso em: 26 set. 2005. 
30 PASSOS, L. M. L.; PARK, Y. K. Fructooligosaccharides: implications in human health being and use in foods. Ciência Rural, v.33, n.2, p.385-390, 2003.

31 SANDERS, D. J.; OMES, D.; EARL, L.K. The absorption, distribution and excretion of one and two (C) palmitoyl triacyglycerols in the rat. Journal Food and Chemical Toxicology, v.39,p.709-716,2001.

32 SANDROU, D.K; ARVANITOYANNIS, I.S. Low-fat/Calorie Foods: current state and perspectives. Critical Review in Food Science and Nutrition, v. 40, n. 5, p. 427-447, Sep. 2000.

33 SIVIERI, K.; OLIVEIRA, M. Avaliação da vida-de-prateleira de bebidas lácteas preparadas com "fat replacers" (Litesse e Dairylo). Ciência e Tecnologia de Alimentos, v.22, n.1, p. 24-31, 2002.

34 SLESINSKI, M.J.; SUBAR, A.F.; KAHLE, L.L. Trends in use of vitamin and mineral supplements in the United States: the 1987 and 1992 National Health Interview Surveys. Journal of American Dietetic Association, v. 95, n.8, p. 921-923, 1995.

35 THEBAUDIN, J. Y.; LEFEBEVRE, A C. Dietary fibres: nutritional and technological interest. Trends Food Science and Technology, v.8, p.41-48, 1997.

36 TORRES, E. A.F. S. Alimentos do milênio: a importância dos transgênicos, funcionais e fitoterápicos para a saúde. São Paulo: Signus Editora, 2002. p 94.

37 UIEARA, M. Fake fats: a química dos produtos fat free. Revista QMCWEB, n. 24, 2000. Florianópolis, UFSC. On line: www.qmc.ufsc.br/qmcweb/exemplar24.html . Acesso em: 26 set. 2005.

38 WILLIANS, P.A. Gums and stabilizers for the food industry. Cambridge: Royal Society of Chemistry, 2000. 470 p.

39 ZAMBRANO, F.; CAMARGO, C.R.O. Amido de mandioca 
modificado comparado com substitutos de gordura comerciais.

Brazilian Journal of Food Technology, v. 72, n. 4, p.147-154, 2001. 